\title{
Application of Cellular Automata Simulations to Modelling of Dynamic Recrystallization
}

\author{
Jiří Kroc \\ West Bohemia University, Research Center, Univezitní 8, \\ CZ 332 03, Plzeň, Czech Republic, \\ krocj@ntc.zcu.cz, \\ WWW home page: http://home.zcu.cz/ ${ }^{\mathrm{krocj} /}$
}

\begin{abstract}
The cellular automaton computer simulation technique has been applied to the problem of dynamic recrystallization. The model is composed of simple microstructural mechanisms - such as evolution of dislocation density, grain boundary movement and nucleation - that acting together result in complex macrostructural response expressed by evolution of flow stress and grain size. A strong sensitivity of the flow stress curves to the initial grain size and nucleation rate is observed. Simulations lead to the conclusion that model of a dynamically recrystallizing material has a strong structural memory resulting in wide variety of experimentally observed responses.
\end{abstract}

\section{Introduction}

The plastic deformation of metals and alloys at homologous temperatures higher than 0.5 leads to two types of behaviour [7]. For the first type - i.e., for the high stacking fault energy materials - a dynamic equilibrium between the generation and accumulation of dislocations on one side and annihilation with rearrangement of dislocations on the other side is reached. In this way, a constant flow stress is approached after a certain amount of strain. Therefore, no dynamic recrystallization (DRX) is observed.

For the second type of behaviour - i.e., for medium and low stacking fault energy materials - a higher storage rate is reached due to lower rates of dynamic recovery. At the same time, small dislocation-free regions are produced by dislocation rearrangement at grain boundaries. These small dislocation-free regions - called nuclei - can grow into the surrounding material that contain dislocations. Such recrystallized regions are separated from the surrounding material by high angle boundaries. This process is called dynamic recrystallization where the word dynamic indicates that recrystallization operates simultaneously with deformation.

The flow stress curves of a metal subjected to DRX exhibit the single peak behaviour at low temperatures or high strain rates, and the multiple peak behaviour. Multiple peak behaviour is typically dumped towards a steady state value, at high temperatures or low strain rates. Grain growth is either deformation controlled at higher strain rates that results in fine grains, or impingement controlled at lower strain rates that results in coarse grains. 
The driving force for grain boundary movement is relatively quickly decreased by the rapid production of dislocations at higher strain rates. The grain boundary velocity is not fast enough to replace the initial structure at such strain rates. Several nucleation waves operate simultaneously, and therefore the single peak on the flow stress curve is observed. There is less reduction in the driving force at lower strain rates. The synchronized recrystallization of the material results in a decrease of stress on the flow stress curve. Further deformation leads to the production of new dislocations that results in an increase of the stress on the flow stress curve until a new nucleation wave is triggered that again results in full recrystallization and a new drop on the flow stress curve. This process results in the multiple peak behaviour.

Sakai et al. [12] have shown that there exists a transition from the multiple to the single peak behaviour for materials in which nucleation operates at grain boundaries, and that this transition occurs at the ratio $D_{0} / D_{s s}=2$, where $D_{0}$ is the initial grain size and $D_{s s}$ is the steady state value. The single peak behaviour occurs when $D_{0} / D_{s s}>2$ and the multiple peak behaviour when $D_{0} / D_{s s} \leq 2$. Sakai et al. carried out strain rate change tests in which an abrupt increase and/or decrease of the strain rate during deformation was applied, as described in [12]. It was observed that, for example, the established multiple peak behaviour could be replaced by the single peak behaviour by an abrupt increase of the strain rate. An excellent review of such DRX experiments can be found in the work of Sakai and Jonas [13].

The Monte Carlo method was applied to model DRX in work of Rollett et al. [11] and Peczak [10]. They predicted the effect of the initial grain size, the steady state grain size dependence on the steady state stress, and the dependence of the peak stress on the peak strain. Unfortunately, they were unable to reproduce the results of the test in which the initial grain size was changed under identical deformation conditions, i.e. the horizontal test.

The Cellular Automata (CA) model of DRX was proposed by Goetz and Seetharaman [4]. They focused their attention on an explanation of flow stress curve shape, the horizontal test and the hardening rate curve, but they did not explain the stress-strain and grain size curves of strain rate change test. They get rather unrealistic grain shapes using growing octagons in their simulations. General information about the CA-paradigm can be found in the the book [2]. It should be mentioned that CA was first time applied to static recrystallization (SRX) by Hesselbarth and Göbel [6].

The objective of the present work is to build a new $\mathrm{CA}$-model where the attention will be focused to several following directions: the grain boundary movement, grain size curves, influence of the initial grain size, horizontal test, influence of nucleation activity, and the strain rate change test.

\section{CA-model of DRX}

The proposed CA-model of DRX is composed of an uniform two-dimensional lattice of cells that are updated simultaneously according to a transition rule. 
The evolution of a cell is controlled by the cells that form the neighbourhood or surrounding of this cell. The Moore neighbourhood, i.e. the eight first and second nearest neighbours in a square lattice, is used with the probabilistic grain boundary movement that produce growing grains of approximately globular shape before their impingement. The lattice size used in simulations is $256 \times 256$ cells. Boundary effects of the lattice are removed by use of periodic boundary conditions. Simply, the upper with the lower edge of the lattice are attached together and the left with the right edge of the lattice are attached together as well.

Every CA-simulation of DRX can be decomposed into two steps. In the first one, the initial $\mathrm{CA}-$ microstructure is produced. The initial $\mathrm{CA}$-microstructure is generated by use of growth of randomly generated nuclei in homogeneous medium, i.e. by simulation of SRX. The following simple transition rule is used. A cell under consideration recrystallize with $50 \%$ probability if at least one of the neighbours from the Moore neighbourhood is recrystallized. Therefore, growing grains reach approximately globular shape.

In the second step, the simulation of DRX is done by the sequential realization of the following three steps representing microstructural evolution of each cell during each time step: (a) evolution of the dislocation density, (b) recrystallization realized by the growth of grains when driving force exceeds a critical value, and (c) the nucleation of embryos of new grains. Three variables, defined for every cell separately, are used in the $\mathrm{CA}$-model: the dislocation density $\rho$, the orientation $\theta$, and the waiting time $t_{w}$, affecting the grain boundary migration velocity.

The dependence of the dislocation density $\rho$ of one particular cell on the shear strain $\gamma$ is expressed by the following law

$$
\frac{d \rho}{d \gamma}=A \rho^{1 / 2}-B \rho
$$

which is the Mecking and Kocks law [9] at the macroscopic level, where $A$ and $B$ are constants. The first term represents strain hardening and the second term the recovery of dislocations. The flow stress $\sigma$ is proportional to the square root of $\bar{\rho}$, i.e. $\sigma \propto(\bar{\rho})^{1 / 2}$, where the average dislocation density $\bar{\rho}=\left(\sum_{i j} \rho_{i j}\right) / 256^{2}$.

It is considered that 18 different orientations are present in the plane. The velocity of a moving grain boundary can be expressed by the temperature $T$ dependent waiting time $t_{w}(T)$, as $v(T)=d /\left(t_{w}(T)+2\right)$, where $d$ is the cell width.

A recrystallization event will occur at a cell $C$ under consideration with $50 \%$ probability when the following conditions are fulfilled simultaneously: (i) the cell $C$ is situated at a grain boundary (GB), (ii) the difference in dislocation density between the cell $C$ and neighbouring cell belonging to different grain is greater than a critical value $\Delta \rho_{c r}$, (iii) the potential new configuration of the GB is not an excluded one (e.g. one cell wide re-entrant bulges are not allowed), (iv) the waiting time $t_{w}$ is zero, (v) a grain having lower dislocation density grows into a grain having a higher dislocation density. 
When there is no recrystallization event for the cell $C$ under consideration at the given time $t$, then the following steps are taken: the waiting time $t_{w}$ is decreased by one, and the dislocation density $\rho$ follows equation 1 .

Finally, a nucleus is produced randomly at the GB with a probability of $0.1 \%$ if the dislocation density exceeds a value $\rho_{\text {nucl }}$. The value $\rho_{n u c l}$ is kept constant, because there is assumed no temperature the CA-model, except the case when a dynamic change of nucleation rate is applied. The production of a nucleus sets the dislocation density $\rho$ to zero, waiting time $t_{w}$ is set to its maxiaml value, and the new and different orientation $\theta$ of the nucleus is chosen randomly from all possible orientations.

\section{Results and Discussion}

The initial CA-microstructure of the DRX simulations (cf. Fig. 3 (a)) was produced using an improved Hesselbarth-Göbel algorithm [6], i.e. by the simulation of SRX. This leads to the globular shape of the grains at any time in a CAsimulation (cf. Fig. 3 (a)) instead of the octagonal shapes as in the work of Goetz and Seetharaman [5] or the rectangular shapes in the work of Hesselbarth and Göbel [6] and of other authors. Octagonal grain shapes were also used by Goetz and Seetharaman [4] in their CA-simulations of DRX.

\subsection{Flow Stress and Grain Size Curves}

DRX experiments carried out under constant strain rate and temperature are called constant strain rate tests. Those experiments displays the transition of flow stress curves from the multiple peak mode - called by some authors cyclic or oscillations - to the single peak mode of recrystallization if different temperature and/or strain rate are taken. The transition is passed by increasing the strain rate or decreasing temperature of the whole test. Sakai, Jonas and co-workers (cf. [13] and references therein) started to pay more attention to the microstructural processes in metals undergoing DRX. Beside the other results, these investigations led to the conclusion that the single peak behaviour is associated with grain refinement and the multiple peak behaviour is associated with several distinct cycles of grain coarsening [13].

Flow stress curves for two initial grain sizes $\bar{D}_{0}=2.9$ (solid lines) and 7.0 (dashed lines) simulated by the proposed CA-model of DRX are presented in Fig. 1 (a). As the complementary information, a set of grain size curves is displayed in Fig. 1 (b) for the same initial grain sizes $\bar{D}_{0}=2.9$ (solid lines) and 7.0 (dashed lines). The multiple or single peak behaviour is produced at high or low temperature, respectively (what is consistent with experimental results [14]). The peak stress $\sigma_{p}$ is shifted upwards and to the right with an increase of the initial grain size. Two CA-microstructures are given in Fig. 3: the initial one (Fig. 3 (a)), and that corresponding to the first grain size minimum (Fig. 3 (b), curve marked by the letter $m$ ). Refinement occurs before the first minimum is reached which is replaced by coarsening until the first maximum (cf. Fig. 1 (b)). 

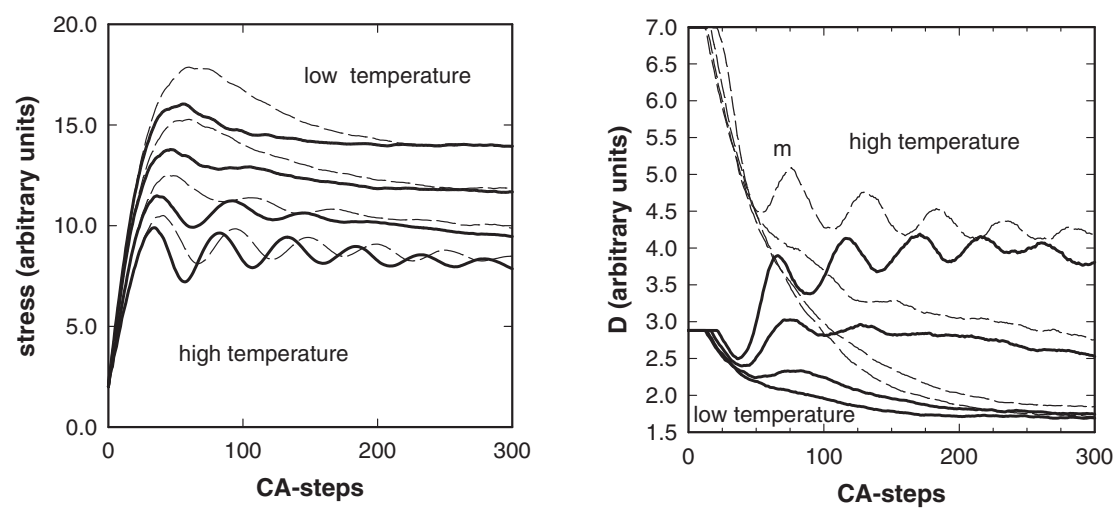

Fig. 1. (a) Flow stress curves for four different temperatures, representing deformation at a constant strain rate and for two different initial grain sizes $\bar{D}_{0}=2.9$ (solid lines) and 7.0 (dashed lines). (b) Dependence of grain size CA-step for the same deformation conditions as in figure (a) and for the same two initial grain sizes where the symbol $\bar{D}$ has been replaced by the symbol $D$. (The figure (a) is always on the left.)

It is observed during $\mathrm{CA}$-simulations - in the CA-microstructure undergoing deformation - that the first nuclei are produced before the peak stress is reached. These nuclei grow, in the case of the multiple peak behaviour, almost in synchronism and they produce a recrystallization wave resulting in a stress decrease on the flow stress curve. The entire CA-microstructure is consumed by recrystallization after a certain amount of time, then recrystallization is terminated. Continuing accumulation of deformation gradually increases stress. The whole cycle is repeated after the production of new generations of nuclei again and again. The multiple peak behaviour is observed in this way. A decrease in the degree of synchronization of the above mentioned nucleation waves leads to the gradual disappearance of the oscillations on the flow stress curves during the multiple peak behaviour.

Several overlapping nucleation waves are produced at the beginning of recrystallization during the single peak behaviour. These overlapping waves are not synchronized, and therefore, they produce one sole peak on the flow stress curve. These characteristics of the single and the multiple peak behaviour are consistent with the experimental work of Sakui et al. [14].

Multiple peaks are observed on grain size curves as well (cf. Fig. 1 (b)). Minima and maxima of the grain size curve are phase shifted by approximately $1 / 4$ of a period before the corresponding maxima and minima of the flow stress curve during the multiple peak behaviour. This phase shift is consistent with MC-simulations $[11,10]$. On the other side, the experimental curves displaying evolution of grain size do not exhibit multiple peaks in the case of the multiple peak behaviour. The reason of this discrepancy could lay in the fact that exper- 
imental results are not in situ measurements but post mortem one (cf. details of [14]).

\subsection{Sensitivity of Material Response to the Initial Grain Size}

A strong influence of the initial grain size on the shape of stress-strain curves is experimentally well documented $[13,14]$. As discussed earlier, it is well known that the transition from the multiple to the single peak behaviour can be passed by mere change of the initial grain size in a set of tests where deformation conditions - i.e., strain rate and temperature - are kept constant.

On one hand, there are known materials where all the first peak stresses of every curve in a horizontal test are almost identical (for example steel [13]). On the other hand, there are materials where the first peak stresses of the single peak behaviour is significantly shifted upwards compared to the peak stresses of the multiple peak behaviour (cf. copper [1]).

It should be mentioned here that the Monte Carlo simulations do not succeed to explain this type of transition $[11,10]$. On the other side, CA-simulations successfully explained it [4]. In this and one subsequent section, it will be studied which other mechanisms can cause a shift of the peak stress of the single peak curve compared to the first peak stress of the multiple peak curve.

In the present simulations, it is evident from Figs. 1 and 2 that the initial grain size $\bar{D}_{0}$ has a strong influence on the shapes of the flow stress and grain size curves for given deformation conditions. It is observed that more coarse $\bar{D}_{0}$ values shift the flow stress curve upwards and result in higher peak stresses $\sigma_{p}$ at the same time (cf. Fig. 1). The steady state value is not influenced by any change in $\bar{D}_{0}$ (cf. Fig. 1 (a) and 1 (b)). This means that a metal subjected to DRX reaches the same dynamically stable equilibrium for the same deformation conditions from any initial grain size $D_{0}$.

The transition from the single to the multiple peak behaviour in a horizontal test - where the strain rate, temperature and nucleation rate are kept constant - is reached for the ratio of initial grain size $\bar{D}_{0}$ to steady state grain size $\bar{D}_{s s}$, $\bar{D}_{0} / \bar{D}_{s s}=3.5 \pm 0.5$. This is somewhat higher value than the experimental ratio $\bar{D}_{0} / \bar{D}_{s s}=2$. It has to be stressed here that the experimentally derived transition ratio is just an approximate value and not the precise one (cf. details [13]).

It is well known from experiments that grain coarsening is associated with multiple peak behaviour, and that grain refinement is associated with single peak if the final grain size is less than half of the initial one. Several different inverse transition ratios $\bar{D}_{s s} / \bar{D}_{0}$ are observed in simulations. Inverse transition ratio of $\bar{D}_{s s} / \bar{D}_{0}=0.3$ is observed if the initial grain size is changed only. Different inverse transition ratios can be reached when nucleation rate is simultaneously changed during simulation.

\subsection{Strain Rate Change Test}

A special type of experiments that combine a constant strain rate test and an abrupt strain rate increase/decrease test - both during one experiment - will 

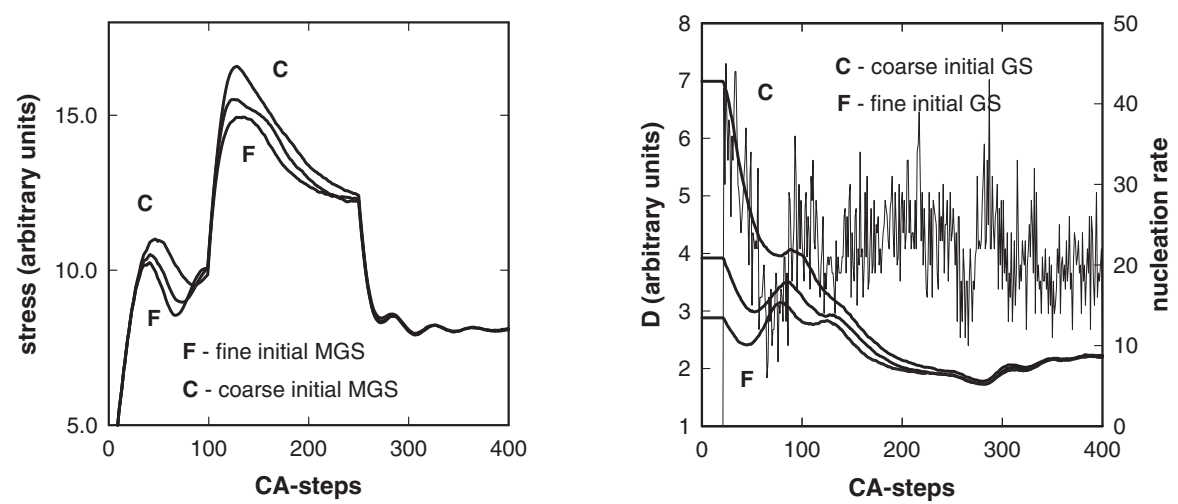

Fig. 2. (a) Flow stress curves in the case of the strain rate jump test for three different initial grain sizes $\bar{D}_{0}=2.9$ (curve F), 3.9 and 7.0 (curve C) carried out under identical deformation conditions. The sensitivity of the flow stress curves to the initial value of $\bar{D}_{0}$ is apparent. (b) Dependence of grain size curves on the CA-step for the same deformation conditions. The thin curve represents the nucleation rate $\dot{N}$ associated with the curve marked by the letter $F$.

be briefly introduced here. In this type of experiments, first more or less stable mode of behaviour for a given constant strain rate and temperature is reached, and then an abrupt change of strain rate is applied. Strain rate increase or strain rate decrease are two possible changes during such experiments. A combination of several abrupt strain rate changes is usually applied during one experiment (cf. details in [13]). Typically, the strain rate was cycled from a low value to a high value and back to the initial value.

One particular case of a simulated strain rate change test is shown in Fig. 2, where an initial strain rate was abruptly increased to a higher strain rate after $100 \mathrm{CA}$-steps, and abruptly decreased to the original strain rate after another $150 \mathrm{CA}$-steps. Three different initial grain sizes, $\bar{D}_{0}=2.9,3.9$ and 7.0 , led to three different flow stresses and grain size curves. The peak stress $\sigma_{p}$ is shifted upwards when the initial grain size $\bar{D}_{0}$ is coarsened (Fig. 2 (a)). The transition from the multiple to the single peak behaviour and vice versa is reproduced (compare to experimental results [12]). Such transition can be interpreted as switching from one mode of behaviour to the other. This transition is consistent with experimental results of Sakai et al. [12]. They showed that a steel deformed at a given elevated temperature, for which the strain rate was cycled from a low value to a high value and back to the initial value, exhibits a transition from the multiple to the single peak behaviour and vice versa.

The evolution of grain size $D_{0}$ presented in Fig. 2 (b) is consistent with the experiments [12], in which high or low strain rate is associated with fine or coarse steady state grain size $\bar{D}_{s s}$, respectively. Details can be found in the 

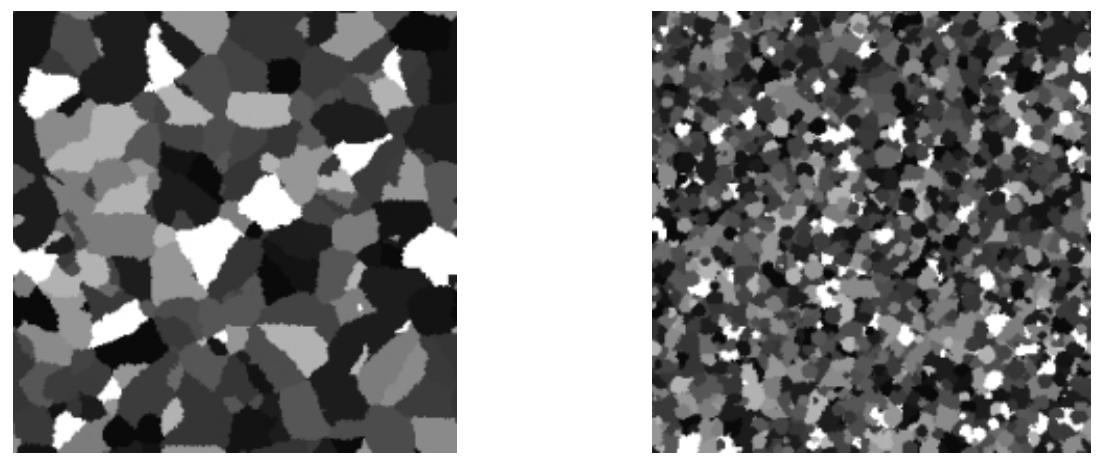

Fig. 3. Two CA-microstructures corresponding to two different $\mathrm{CA}-$ steps in the case of the multiple peak behaviour for the curve of grain size dependence on the CA-step identified by the letter $m$ in Fig. 1 (b): (a) the initial CA-microstructure, (b) the $\mathrm{CA}-$ micrograph is taken at the first minimum. Grain refinement is apparent.

microstructural mechanism map distinguishing these two modes of DRX [12]. This and the previous simulations leads to the conclusion that a dynamically recrystallizing material has a strong structural memory resulting in a wide range of experimentally observed responses. Strain rate decrease tests or more complex tests can be easily realized by such $\mathrm{CA}-$ model as well.

It is worth to note that instabilities were detected in some specific cases during the simulations. These instabilities can produce "spirals" in the CAmicrostructures, typically after a strain rate decrease during a strain rate test. These spirals affect the shapes of the flow stress and grain size curves. The occurrence of such spirals could be associated with the assumption of the CAmodel that every cell is deformed at the very same strain rate and that there are no deformation localizations in the $\mathrm{CA}$-microstructure.

The evolution of the nucleation rate is shown in Fig. 2 (b) by the thin curve. The nucleation rate $\dot{N}$ is simply defined as the number of nuclei per time step. It was recognized that the nucleation rate $\dot{N}$ is inversely correlated with the grain size curve. This correlation occurs because finer grains are associated with higher specific grain boundary areas and therefore with higher nucleation rates as long as only GB nucleation is allowed. Thus, an increase in the nucleation rate $\dot{N}$ is correlated with a decrease in the grain size $\bar{D}$ and vice versa.

\subsection{Dynamic Change of Nucleation Rate}

The influence of different initial nucleation rates on the flow stress curve for the initial grain size of $D_{0}=9.8$ can be seen in Fig. 4 where different nucleation rates are taken for the frst $50 \mathrm{CA}$-steps. Nucleation rate is changed from an initial value to the fixed value of 0.001 where the initial value is equal to $0.0001,0.001,0.002,0.01$ from top to bottom, i.e. $A, B, C, D$, respectively. The curve marked as $O$ is computed for different initial grain size of $D_{0}=2.8$ 
without change of nucleation rate equal to 0.001 . The ratio of the initial grain size $\bar{D}_{0}$ to the steady state grain size $\bar{D}_{s s}$ is $\bar{D}_{0} / \bar{D}_{s s}=3.9 \pm 0.4$.

The ratio of the initial grain size $\bar{D}_{0}$ to the steady state grain size $\bar{D}_{s s}$ gives $\bar{D}_{0} / \bar{D}_{s s}=2.8 \pm 0.4$ where the initial grain size of $D_{0}=7.0$ is taken. The lower limit of this ratio lays near of the experimentally observed value of 2 for which the inverse transition ratio $\bar{D}_{s s} / \bar{D}_{0}$ equal to 0.4 approaches the experimental value of 0.5 . Those observations lead to the conclusion that nucleation rate can affects the shape of flow stress curves beside the initial grain size. Different thermomechanical histories of a material can lead to a different initial nucleation rates resulting in different shapes of deformation curves.
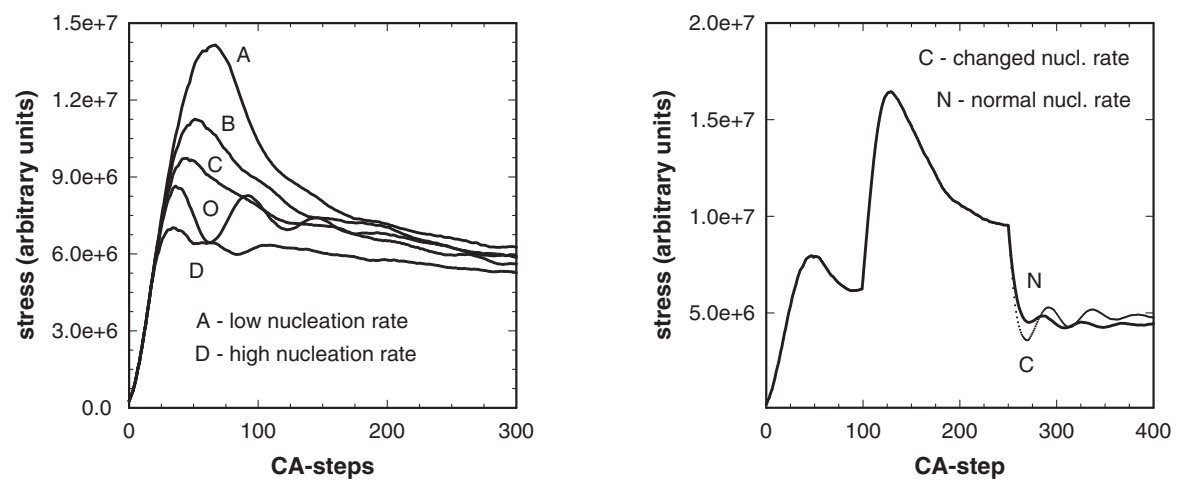

Fig. 4. The influence of different initial nucleation rates on: (a) the flow stress curves for the initial grain size of $D_{0}=9.8$ with change of the nucleation rate for the first $50 \mathrm{CA}$-steps. The nucleation rate is changed from an initial value to the fixed value of 0.001 where the initial value is equal to $0.0001,0.001,0.002,0.01$ from top to bottom, i.e. $A, B, C, D$, respectively; (b) strain rate change test at the point of strain rate decrease. Temporary increase of nucleation rate pronounce oscillations after strain rate decrease.

The influence of nucleation rate change on the shape of deformation curve during strain rate change test has been studied, see Fig. 4 (b). Solid line represents the same strain rate change test as in Fig. 2 (cf. curve C), i.e. the situation where the nucleation rate is held at constant value of 0.001 . The dotted line represents the situation when the nucleation rate is increased for $20 \mathrm{CA}$-steps from 0.001 to 0.01 at the $\mathrm{CA}$-step equal 250 - right at the moment of an abrupt strain rate decrease - and after this number of CA-steps decreased to its original value. It is evident that such change of nucleation rate can pronounce the maxima and minima on deformation curve.

\section{Conclusions}

A cellular automaton (CA) model of dynamic recrystallization (DRX) has been proposed with a probabilistic movement of the grain boundaries. The $\mathrm{CA}$-model 
itself is simple to define and easy to handle. A strong influence of the initial grain size and nucleation rate on the shape of flow stress curves is observed. It is found that the evolution of grain size provides information that is complementary to the flow stress curve. Changes of nucleation rate shifts the maxima of the flow stress curves and transition from the multiple peak to the single peak mode. It is recognized that the material has a strong structural memory and selforganizes itself into dynamically stable modes of behaviour. Those dynamically stable modes are clearly marked on the deformation curves and grain size curves by the steady state values.

\section{Acknowledgements}

The author is greatly indebted to Prof. J.D. Eckart for his publicly available $\mathrm{CA}-$ environment called Cellular/Cellang [3] that have been used to compute results presented in this work. The author acknowledge financial support from the Czech Ministry of Education under project LN00B084.

\section{References}

1. Blaz, L., Sakai, T., Jonas, J.J.: Effect of initial grain size on dynamic recrystallization of cooper. Metal Sci. 17 (1983) 609-616

2. Chopard, B., Droz, M.: Cellular Atomata Modeling of Physical Systems. Cambridge University Press, Cambridge (1998)

3. Eckart, J.D.: The Cellular Automata Simulation System: Cellular/Cellang (19911999) http://www.cs.runet.edu/ dana/

4. Goetz, R.L., Seetharaman, V.: Modelling Dynamic Recrystallization Using Cellular Automata. Scripta Mater. 38 (1998) 405-413

5. Goetz, R.L., Seetharaman, V.: Static Recrystallization Kinetics with Homogeneous and Heterogeneous Nucleation Using a Cellular Automata Model. Metall. Mater. Trans. A 29 (1998) 2307-2321

6. Hesselbarth, H.W., Göbel, I.R.: Simulation of Recrystallization by Cellular Automata. Acta Metall. Mater. 39 (1991) 2135-2143

7. Humphreys, F.J., Hatherly, M.: Recrystallization and Related Annealing Phenomena. Pergamon, Oxford, New York, Tokyo, 1996

8. Kroc, J.: Simulation of Dynamic Recrystallization by Cellular Automata. PhD Thesis. Charles University, Prague, 2001

9. Mecking, H., Kocks, U.F.: Kinetics of flow and strain-hardening. Acta. Metall. 29 (1981) 1865-1875

10. Peczak, P.: A Monte Carlo study of influence of deformation temperature on dynamic recrystallization. Acta Metall. Mater. 43 (1995) 1279-1291

11. Rollett, A.D., Luton, M.J., Srolovitz, D.J.: Microstructural Simulation of Dynamic recrystallization. Acta Metall. Mater. 40 (1992) 43-55

12. Sakai, T., Akben, M.G., Jonas J.J.: Dynamic recrystallization during the transient deformation of a vanadium microalloyed steel. Acta Metall. 31 (1983) 631-642

13. Sakai, T., Jonas, J.J.: Dynamic recrystallization: mechanical and microstructural considerations. Acta. Metall. 32 (1984) 189-209

14. Sakui, S., Sakai, T., Takeishi, K.: Hot deformation of austenite in a plain carbon steel. Trans. Iron Steel Inst. Japan 17 (1977) 718-725 